\title{
Mechanism of Resistance to Macrolide-Lincosamide-Streptogramin Antibiotics in Streptococcus thermophilus
}

\author{
George A. Somkuti ${ }^{1}$, John A. Renye ${ }^{1} \&$ Dennis H. Steinberg ${ }^{1}$ \\ ${ }^{1}$ Eastern Regional Research Center, Agricultural Research Service, U.S. Department of Agriculture, 600 East \\ Mermaid Lane, Wyndmoor, Pennsylvania 19038, USA
}

Correspondence: George A. Somkuti, United States Department of Agriculture, Wyndmoor, PA 19038, USA. Tel: 1-215-233-6474. E-mail: george.somkuti@ars.usda.gov

Received: October 5, 2014 Accepted: October 28, 2014 Online Published: November 6, 2014

doi:10.5539/jfr.v4n1p58 URL: http://dx.doi.org/10.5539/jfr.v4n1p58

Mention of trade names or commercial products in this publication is solely for the purpose of providing specific information and does not imply recommendation of endorsement by the U.S. Department of Agriculture. USDA is an equal opportunity provider and employer.

\begin{abstract}
Resistance to macrolide-lincosamide-streptogramin (MLS) group antibiotics in the dairy bacterium Streptococcus thermophilus (ST) is documented but the mechanism of resistance has not been elucidated. MIC values for erythromycin (Erm), azithromycin (Azm), tylosin (Tyl), spiramycin (Spm), pristinamycin (Prm) and virginiamycin S (Vir) were determined by the disk diffusion method. PCR products were obtained with primer pairs for the L4, L22 and 23S rDNA (domain V) genes. The sequencing results ruled out mutations in the L4 and L22 ribosomal proteins and the presence of rRNA methylase, efflux, and inactivating genes. However, sequencing of domain $\mathrm{V}$ in each of the six ribosomal alleles detected by EcoRI/I-CeuI digestion in ST mutants identified three types of mutations that led to MLS resistance. Type A mutants, induced by Erm, had high resistance to 14- and 15-membered ring macrolides (Erm, Azm) and streptogramin B antibiotics (Prm, Vir), moderate resistance to 16 -membered ring macrolides (Tyl, Spm), but remained susceptible to lincomycin. In Type B mutants, also induced by Erm, resistance was high to Erm, Tyl and Spm, and moderate to lincomycin but sensitivity was retained to Prm. Type C mutants, induced by Prm, showed high resistance to 16-membered ring macrolides but remained sensitive to Erm, Azm and lincomycin. The three identifiable resistance patterns were apparently due to point mutations in domain $\mathrm{V}$ of the $23 \mathrm{~S}$ rRNA gene, resulting in three phenotypes among resistant $S$. thermophilus isolates. Type A phenotype mutants had a C2611 G mutation in five of the six ribosomal alleles, Type B phenotypes had a A2058G mutation in five alleles, and Type C variants had a A2062C mutation in all six alleles. Resistance to MLS antibiotics in S. thermophilus was inducible by 14- and 15 -membered ring macrolides and streptogramin B type antibiotics but not by 16-membered ring macrolides or lincosamides.
\end{abstract}

Keywords: macrolide antibiotic resistance, Streptococcus thermophilus

Abbreviations: ST-Streptococcus thermophilus; Erm-erythromycin; Azm-azithromycin; Tyl-tylosin; Spm-spiramycin; Prm-pristinamycin; Vir-virginiamycin; Cli-clindamycin; MLS-macrolide-lincosamide-streptogramin

\section{Introduction}

Streptococcus thermophilus is a widely used food-grade lactic acid bacterium responsible for carrying out essential biocatalytic functions in the industrial production of yogurt and other fermented dairy foods, including Italian and Swiss style cheeses. Previously, several reports identified S. thermophilus as a potential carrier of antibiotic resistance determinants, including MLS antibiotic resistance genes (Sozzi \& Smiley, 1980; Wang et al., 2005; Tosi et al., 2007). Since dairy fermentations also provide a suitable environment for the growth of pathogenic bacteria, there has been a persistent concern about the possible interchange of antibiotic resistance genes between S. thermophilus and pathogens that may proliferate in the human gastrointestinal tract. Although the erythromycin resistance phenotype in $S$. thermophilus is documented in the literature, the mechanism of 
resistance to antibiotics of the macrolide-lincosamide-streptogramin (MLS) group in this important industrial species has not been studied at the molecular level.

In clinical isolates of streptococci, resistance to macrolide antibiotics is orchestrated predominantly by two well-known resistance mechanisms, target site modification and macrolide-specific efflux. In target site modification, an acquired ermB gene specifically methylates A2058 in 23S rRNA and blocks the binding of macrolides by causing conformational changes in the $50 \mathrm{~S}$ ribosomal subunit, resulting in cross-resistance to 14-(erythromycin), 15-(azithromycin), and 16-membered (tylosin) macrolides. In macrolide-specific efflux that is mediated by a membrane protein encoded by the mef $(\mathrm{A})$ gene, resistance develops to 14- and 15-membered macrolides but not to 16-membered macrolides, lincosamides and streptogramins (Depardieu \& Courvalin, 2001). Other known macrolide resistance mechanisms involve mutations in the ribosomal proteins L4 and L22, and also the 23S rRNA (Tait-Kamradt et al., 2000; Pihlajamaki et al., 2002).

In this study, the mechanism of resistance to MLS antibiotics was elucidated in mutants of S. thermophilus ST113 displaying different phenotypes that were isolated following exposure to macrolide antibiotics.

\section{Methods}

\subsection{Bacterial Strains and Induction of MLS Resistance}

The identity of S. thermophilus strain ST113 (deposited as NRRL B-59386 in the Agricultural Research Service Culture Collection, NCAUR-USDA, Peoria, IL, http://nrrl.ncaur.usda.gov) was confirmed by 16S rRNA gene sequence analysis (MIDI Labs, Inc., Newark, DE). The culture was maintained in tryptone-yeast extract-lactose (TYL) medium at $37^{\circ} \mathrm{C}$ (Somkuti \& Steinberg, 1986). MLS resistance was induced out by growing ST113 on TYL agar plates supplemented with 14-membered lactone ring (erythromycin, Sigma Chemical Co., St. Louis, MO), 15-membered lactone ring (azithromycin, Pfizer Inc., New York, NY) or 16-membered lactone ring (spiramycin and tylosin, Rhone-Poulenc Rorer, Paris, France) macrolide antibiotics, streptogramin B-type antibiotics (pristinamycin and virginiamycin S, gifts from B. Weisblum, University of Wisconsin, Madison), or lincosamide antibiotics (clindamycin and lincomycin, Sigma Chemical Co.), using each at $15 \mu \mathrm{g} \mathrm{mL}^{-1}$. After incubation for $72 \mathrm{~h}$ at $37^{\circ} \mathrm{C}$, single colonies were picked into TYL/antibiotic broth and passaged daily for 3 days before plating serially diluted cultures on TYL/antibiotic agar plates. Antibiotic sensitivity of isolates was evaluated by the disk diffusion assay method.

\subsection{Detection of MLS Resistance Phenotypes}

The resistance patterns in ST113 mutants was detected by a paper disk-agar diffusion technique. This was done by applying $150 \mu \mathrm{L}$ of each antibiotic stock solution $\left(1,00 \mu \mathrm{g} \mathrm{mL}^{-1}\right)$ to filter paper disks and placing them in a grid pattern on agar films. Each plate was inoculated with $2 \times 10^{6} \mathrm{cfu} \mathrm{mL}^{-1}$ cells of ST113 cultures that were grown from single cfu-s isolated following exposure to MLS antibiotics. Plates were incubated at $37^{\circ} \mathrm{C}$ for $24 \mathrm{~h}$.

\subsection{Determination of MIC Values}

The minimum inhibitory concentration (MIC) of each antibiotic was tested by a paper disk agar diffusion method. Samples of serial twofold dilutions of antibiotics (final concentrations from 0.2 to $1,000 \mu \mathrm{g} \mathrm{mL}^{-1}$ were soaked into sterile paper disks and placed on the surface of plates inoculated with putatively identified phenotypic mutants of ST113.

\subsection{Detection of MLS Resistance Determinants}

PCR amplification was used to detect the presence of erm $\mathrm{B}$ and mefA genes in mutant S. thermophilus clones. The primers for erm $\mathrm{B}$ were 5'-GAAAAGGTACTCAACCAAATA-3'(F) and 5'-AGTAACGGTACTTAAATTTAC-3'(R), whereas mefA was detected with the primers 5'-AGTATCATTAATCACTAGTGC-3'(F) and 5'-TTCTTCTGGTACTAAAAGT-GG-3'(R) (Sutcliffe et al., 1996). Mutations in the L4 and L22 ribosomal proteins that also cause macrolide resistance in clinical streptococci were detected with primer pairs described previously (Canu et al., 2002).

PCR was performed with a Perkin-Elmer Thermal Cycler (Model 9700) using the following cycling conditions: $95^{\circ} \mathrm{C}$ for $5 \mathrm{~min}$, followed by 30 cycles of $\left(95^{\circ} \mathrm{C}\right.$ for $1 \mathrm{~min}, 50,55$ or $60^{\circ} \mathrm{C}$ for $1 \mathrm{~min}, 72^{\circ} \mathrm{C}$ for $\left.1 \mathrm{~min}\right)$, followed by 1 cycle of $72{ }^{\circ} \mathrm{C}$ for $7 \mathrm{~min}$ and a $4{ }^{\circ} \mathrm{C}$ soak. PCR products were cleaned up for sequencing using Ampure reagent (Agencourt, www.agencourt.com). Sequencing was performed in an ABI 3730 DNA Analyzer (Applied Biosystems), and sequences were trimmed and aligned using Sequencher software (Genecodes Corp., Ann Arbor, MI). Nucleotide sequences were compared to sequences present in the NCBI database using BLASTn at http://www.ncbi.nlm.nih.gov/. 


\subsection{DNA Sequencing of Ribosomal Alleles}

Genomic DNA was isolated from single clones taken from TYL-agar plates and the number of ribosomal alleles was determined by co-restriction with I-CeuI (New England Biolabs), an intron-encoded enzyme from Chlamydomonas eugametos with a unique 26-bp recognition sequence present only within 23S rRNA genes (Liu et al., 1993), and EcoRI, which cuts outside of 23S rRNA. After I-CeuI/EcoRI co-digestion, DNA fragments were resolved by gel electrophoresis and transferred to nylon membranes using an Oncor Probetech I automated Southern blot system. The probe was a purified 1,003 bp PCR product derived from the 3'end of the 23S rRNA, biotinylated with the BioNick Labeling System (BRL Life Technologies). Hybridization conditions, subsequent washes, streptavidin and phosphatase treatment, and staining with nitrotetrazolium blue and 5-bromo-4-chloro-3-indolyl phosphate were done according to the supplier (Oncor, Inc.).

For sequencing 23S rDNA (domain V) of S. thermophilus (Ludwig et al., 1992), primers were designed using the Genbank sequence (accession number X68429.1) to generate the following primer pairs:

GGTTAAGTTAATAAGGGCGCACGGT(F)

GGAGGCGACCGCCCCAGTC(R)

PCR product: $2271 \mathrm{bp}$

CCTAGCAGTATCCTTTGAGTACGGCG(F)

GGAGGCGACCGCCCCAGTC(R)

PCR product: $1884 \mathrm{bp}$

CCTAGCAGTATCCTGAGTACGGCG(F)

CGCTCCCCAFCACAGCTCAATG(R)

PCR product: $1173 \mathrm{bp}$

CCTAGCAGTATCCTTTGAGTACGGCG(F)

GGATAAGTCCTCGAGCTTATTAGTATTAG(R)

PCR product: $2519 \mathrm{bp}$

\section{Results and Discussion}

\subsection{Induction of Resistance to MLS Antibiotics}

S. thermophilus ST113 colonies appeared within $72 \mathrm{~h}$ on TYL/Erm plates during incubation at $37{ }^{\circ} \mathrm{C}$. A total of $100 \mathrm{cfu}-\mathrm{s}$ were randomly picked for establishing resistance patterns to MLS-type antibiotics, using the grid shown in Figure 1. Based on the results of these bioassays, the Erm resistant mutants were grouped in 2 categories: Type A mutants were highly resistant to Erm, Prm and VirS, with increased resistance to the 16-membered lactone macrolides Spm and Tyl but remained sensitive to Cli and Lin. Type B mutants were similar in resistance to Erm, Spm and Tyl and VirS but remained sensitive to Cli and Lin, as well as Prm.

Incubation of $S$. thermophilus ST113 in the presence of the streptogramin B-type pristinamycin resulted in dozens of cfu-s which uniformly displayed the Type $\mathrm{C}$ resistance pattern: they were highly resistant to the 16-membered lactone ring macrolides Spm and Tyl but remained sensitive to Erm as well as Cli and Lin. Similar results were obtained when ST113 was grown in the presence of VirS.

After exposure to the 15-membered lactone ring Azm, only two resistant clones were present after $72 \mathrm{~h}$. These clones displayed resistance to Spm and Tyl but remained sensitive to Erm, Cli and Lin, as well as to the streptogramin-type Prm (data not shown).

Several attempts to induce resistance in S. thermophilus ST113 with 16-membered lactone macrolides Spm and Tyl were unsuccessful.

\subsection{Molecular Basis for Macrolide Resistance}

Several preliminary experiments were performed to define the molecular basis of the apparently MS phenotype in S. thermophilus ST113. First, the wild-type ST113 was cured of its $4.13 \mathrm{~kb}$ resident plasmid (Somkuti \& Steinberg, 1986) by treatment with ethidium bromide $\left(25 \mu \mathrm{g} \mathrm{mL}{ }^{-1}, 24 \mathrm{~h}\right.$ at $\left.37^{\circ} \mathrm{C}\right)$ ruling out the presence of plasmid borne MLS resistant genes. Screening total DNA extracted from randomly selected clones by Southern blotting with a biotinylated plasmid-based probe yielded several plasmid-free mutants which displayed the same MLS resistance pattern as the wild-type parent culture (Figure 1).

Subsequent screening for the presence of rRNA methylase (ermB) indicated the absence of this resistance mechanism. Since the resistance pattern induced with Erm showed the retention of a Cli and Lin sensitive phenotype, the possible involvement of an active efflux (mefA) gene known to be operational in S. pneumoniae 
and S. pyogenes (Sutcliffe et al., 1996) was tested in S. thermophilus ST113 by PCR techniques. The results of these trials were negative. The absence of mefA involvement in macrolide resistance in ST113 was further confirmed by the addition of the efflux pump inhibitor carbonyl cyanide $m$-chlorophenylhydrazone $(25 \mu \mathrm{M})$ to test media, which failed to influence the outcome of bioassays (Giovanetti et al., 2002).

Next, various domains in the ribosomal architecture were checked for features that are known to be involved in MLS resistance in other species of streptococci. Specific areas encoding L4 and L22 ribosomal proteins were checked for mutations by PCR but DNA sequencing data did not detect any alterations in amplification products. This indicated that the type of macrolide resistance detectable in S. pneumoniae (Canu et al., 2002) was absent in S. thermophilus ST113.

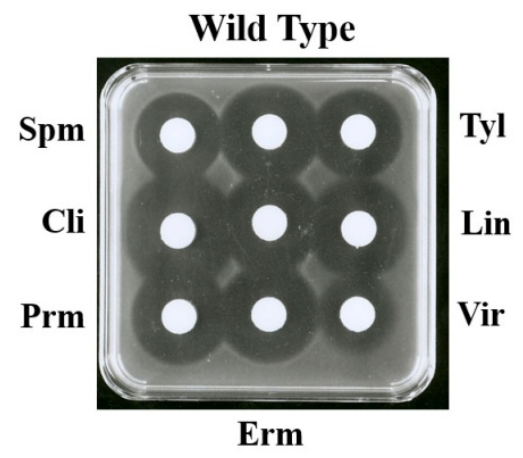

Type B

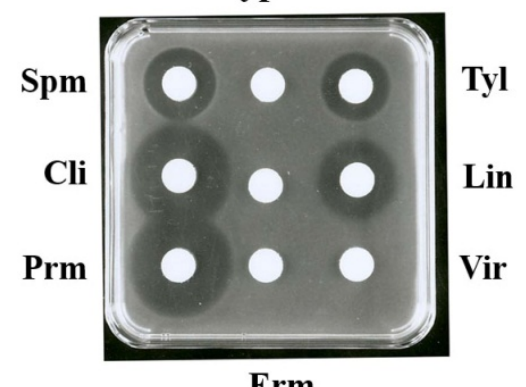

Erm
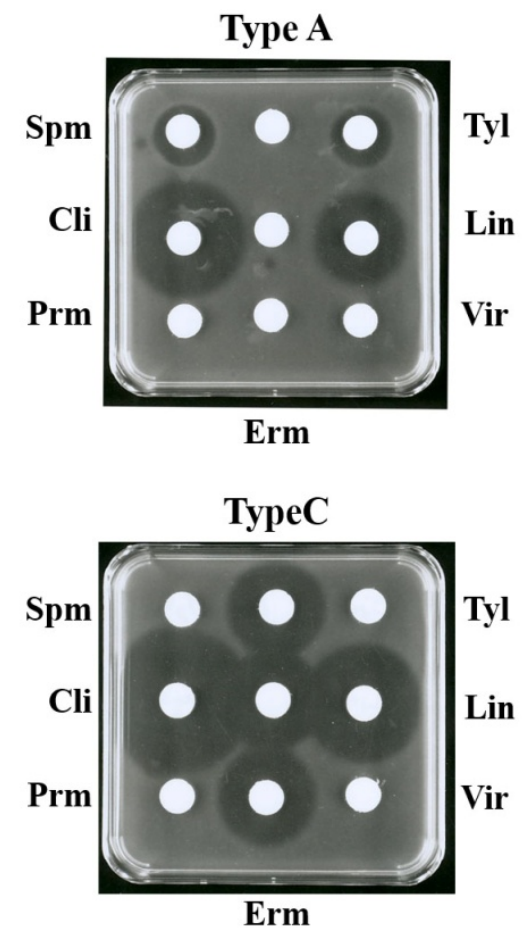

Figure. 1. Resistance patterns detectable in S. thermophilus ST113 mutants after exposure to MLS antibiotics. Each paper disk contained $15 \mu \mathrm{g}$ of antibiotic. Spm: spiramycin, Tyl: tylosin, Cli: clindamycin, Lin: lincomycin, Prm: pristinamycin, Vir: virginiamycin S; Erm: erythromycin

\subsection{Detection of Mutations in Domain V of $23 S$ rRNA}

After ruling out the presence of erm $\mathrm{B}$ and mefA genes as well as mutations in the $\mathrm{L} 4$ and $\mathrm{L} 22$ ribosomal proteins, we targeted domain $\mathrm{V}$ of $23 \mathrm{~S}$ rRNA which is the center of peptidyl transferase activity in the ribosomes and is responsible for the elongation of the nascent peptide chain. Several studies demonstrated that resistance to MLS antibiotics may also develop in streptococci and other bacteria by specific base substitutions in domain $\mathrm{V}$ of $23 \mathrm{~S}$ rRNA (Vester \& Douthwaite, 2001; Pihlajamaki et al., 2002; Jalava et al., 2004; Haanpera et al., 2005).

PCR analysis of genomic DNA from $S$. thermophilus ST113 identified three specific point mutations in $23 \mathrm{~S}$ rRNA, involving nt2058, nt2062 and nt2611 (Figure 2). However, in some cases the exact base calls were ambiguous which indicated that not all ribosomal alleles may have the same base substitutions. To resolve the ambiguity and clearly establish the extent of domain $\mathrm{V}$ involvement in the macrolide resistance phenotype displayed by $S$. thermophilus ST113, the number of ribosomal alleles in the genome was determined. Genomic DNA double-digested with I-CeuI and EcoRI was resolved by AGE and Southern 


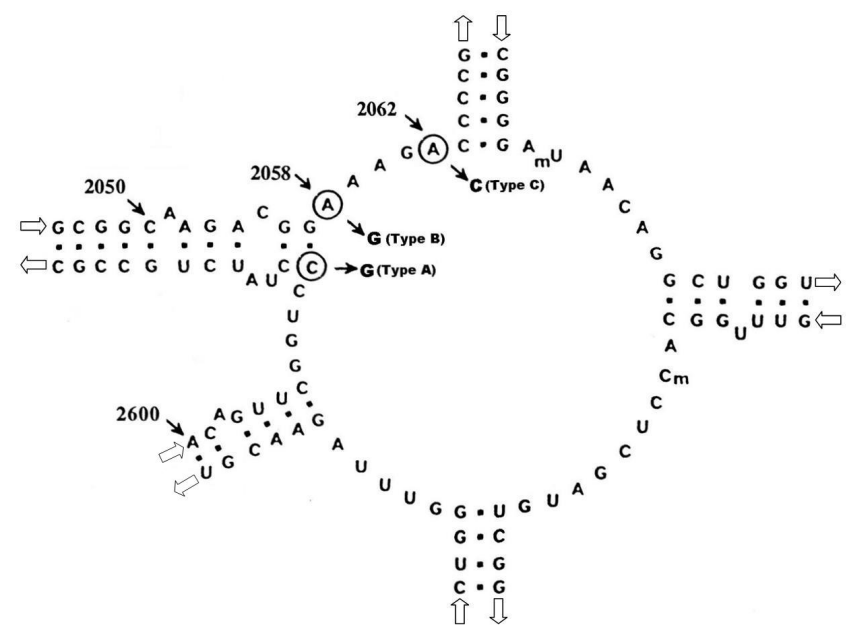

Figure 2. Secondary-structure model of the peptidyl transferase center in domain V of $23 \mathrm{~S}$ rRNA. The circled nucleotides indicate the positions where base substitutions occurred conferring different types of MLS antibiotic resistance patterns in S. thermophilus ST113

hybridyzation tests revealed 6 distinct bands with estimated molecular masses of $23 \mathrm{~kb}, 13 \mathrm{~kb} 9.4 \mathrm{~kb}, 7 \mathrm{~kb}, 6.2$ $\mathrm{kb}$ and $5.2 \mathrm{~kb}$, respectively (Figure 3 ).

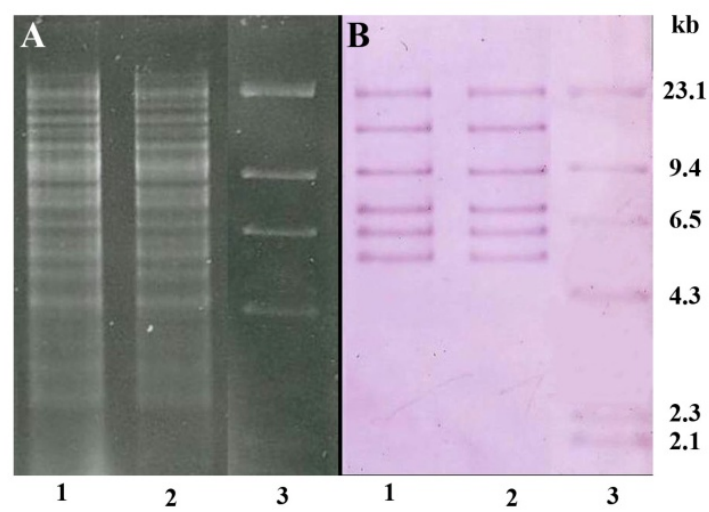

Figure 3. Detection of ribosomal alleles in S. thermophilus ST113. Genomic DNA digested with I-CeuI and EcoRI was resolved by AGE (A). Southern blots with a biotinylated fragment of 23S rRNA as the probe (B); lane 1, MLS-sensitive ST113; lane 2, MLS-resistant ST113; lane 3, MW markers: biotinylated HindIII fragments of $\lambda$ DNA

Bands were extracted from gels and sequenced to determine the exact nature of base substitutions in domain $\mathrm{V}$ of each of the 6 identifiable ribosomal alleles. The results of the DNA sequencing data permitted the identification of three types of domain V mutants (Table 1). Type A phenotype mutants had a specific C2611G mutation in 5 of the 6 ribosomal alleles. Type B phenotype mutants had a A2058G mutation in 5 alleles, while Type $\mathrm{C}$ mutants were homozygous with all 6 ribosomal alleles having a A2062C mutation. A2058G and C2611G mutations are frequently detected in clinical isolates of S. pneumoniae (Vester \& Douthwaite, 2001; Canu et al., 2002; Pihlajamaki et al., 2002). On the other hand, there is one report on a A2062C mutation (Type $\mathrm{C}$ phenotype in ST113) resulting in resistance to 16-membered macrolides (Tyl, Spm) and streptogramins while retaining sensitivity to the 14-membered lactone Erm (Depardieu \& Courvalin, 2001). 
Table 1. Distribution of base substitutions in domain V of six ribosomal alleles in S. thermophilus ST113

\begin{tabular}{|c|c|c|c|c|}
\hline \multicolumn{2}{|c|}{ Alleles } & \multirow{2}{*}{$\begin{array}{l}\text { nt \#2058 } \\
\text { A }\end{array}$} & \multirow{2}{*}{$\begin{array}{l}\text { nt \#2062 } \\
\text { A }\end{array}$} & \multirow{2}{*}{$\begin{array}{l}\mathrm{nt} \mathrm{\# 2611} \\
\mathrm{C}\end{array}$} \\
\hline Wild & & & & \\
\hline \multirow[t]{6}{*}{ Type } & A-1 & A & A & $\mathrm{G}$ \\
\hline & A-2 & A & A & $\mathrm{C}$ \\
\hline & A-3 & A & A & $\mathrm{G}$ \\
\hline & A-4 & A & A & $\mathrm{G}$ \\
\hline & A-5 & A & A & G \\
\hline & A- 6 & A & A & G \\
\hline \multirow[t]{6}{*}{ Type } & B-1 & G & A & $\mathrm{C}$ \\
\hline & B-2 & A & A & $\mathrm{C}$ \\
\hline & B-3 & G & A & $\mathrm{C}$ \\
\hline & B-4 & $\mathrm{G}$ & A & $\mathrm{C}$ \\
\hline & B-5 & $\mathrm{G}$ & A & $\mathrm{C}$ \\
\hline & B-6 & $\mathrm{G}$ & A & $\mathrm{C}$ \\
\hline \multirow[t]{6}{*}{ Type } & C-1 & A & $\mathrm{C}$ & $\mathrm{C}$ \\
\hline & $\mathrm{C}-2$ & A & $\mathrm{C}$ & $\mathrm{C}$ \\
\hline & C-3 & A & $\mathrm{C}$ & $\mathrm{C}$ \\
\hline & C-4 & A & $\mathrm{C}$ & $\mathrm{C}$ \\
\hline & C-5 & A & $\mathrm{C}$ & $\mathrm{C}$ \\
\hline & C-6 & A & $\mathrm{C}$ & $\mathrm{C}$ \\
\hline
\end{tabular}

Site specific mutations resulting in three identifiable phenotypes of $S$. thermophilus ST113 led to substantial changes in the MIC values when the allelic mutants were challenged

with antibiotics. Although Type A and Type B mutants had different base substitutions at different sites in domain $\mathrm{V}$ of the ribosome, both strains showed over a 1,000-fold increase in Erm resistance (Table 2). On the other hand, only the Type A allelic mutant had a substantially increased resistance to pristinamycin and virginiamycin $\mathrm{B}$. Type $\mathrm{C}$ mutants that were induced by exposure to pristinamycin, remained sensitive to Erm but displayed a moderately high level of resistance to the 16-membered macrolides spiramycin and tylosin, while remaining sensitive to the licosamide antibiotics lincomycin and clindamycin.

Table 2. Antibiotic susceptibilities of S. thermophilus ST113 parent and mutant strains

\begin{tabular}{llllllllll}
\hline Strain & \multicolumn{2}{l}{ Site of ribosomal } & \multicolumn{7}{c}{ MIC $\left(\mu \mathrm{gL}^{-1}\right)^{*}$} \\
\hline & mutation & alleles & Erm & Spm & Cli & Pri & Tyl & Lin & Vir \\
\hline Parent & none & none & 0.78 & 6.25 & 0.4 & 12.5 & 3.12 & 1.56 & 12.5 \\
Type A & C2611G & 5 & $>1000$ & 40 & 1.56 & $>1000$ & 18 & 5 & $>1000$ \\
Type B & A2058G & 5 & $>100$ & 400 & 12.5 & 12.5 & 400 & 400 & 400 \\
Type C & A2062C & 6 & 3.12 & 200 & 0.4 & 125 & 750 & 2.5 & 400 \\
\hline
\end{tabular}

* Erm: erythromycin; Spm: spiramycin; Cli: clindamycin; Pri: pristinamycin; Tyl: tylosin; Lin: lincomycin; Vir: virginiamycin $\mathrm{S}$.

The results demonstrated that in the dairy fermentation bacterium S. thermophilus ST113, resistance to macrolide antibiotics and streptogramins but not to lincosamides was inducible with erythromycin (14-member lactone) and azithromycin (15-member lactone) but not with the 16-member lactone macrolides tylosin and 
spiramycin. Streptogramins (pristinamycin and virginiamycin S) induced resistance in S. thermophilus ST113 to 16-member lactone macrolides but not to erythromycin (14-member lactone ring) or lincosamides.

The DNA sequencing data of domain $\mathrm{V}$ in the six identified ribosomal alleles in this bacterium revealed specific point mutations at nt2058, nt2062 and nt2611 that were apparently involved in the resistance mechanism and the display of the three detectable phenotypes. The ribosomal alleles had a heterozygous profile in the case of Type $\mathrm{A}$ and Type B mutants, but were homozygous in mutants displaying a Type $\mathrm{C}$ phenotype.

Since $S$. thermophilus is a widely used bacterium in industrial-scale dairy food production and may come into contact with antibiotic resistant pathogenic streptococci in a production plant environment, further work is needed to elucidate its potential for the possible transmission of antibiotic resistance to other bacteria.

\section{Acknowledgements}

The authors thank C. Briggs for assistance with nucleic acid sequencing.

\section{References}

Canu, A., Malbruny, B., Coquemont, M., Davies, T. A., Appelbaum, P. C., \& Leclercq, R. (2002). Diversity of ribosomal mutations conferring resistance to macrolides, clindamycin, streptogramin and telithromycin in Streptococcus pneumoniae. Antimicrobal Agents and Chemothereapy, 46, 125-134. http://dx.doi.org/10.1128/AAC.46.1.125-131.2002

Depardieu, F., \& Courvalin, P. (2001). Mutation in 23S rRNA responsible for resistance to 16 membered macrolides and streptogramin in Streptococcus pneumoniae. Antimicrobial Agents and Chemotherapy, 45, 319-323. http://dx.doi.org/10.1128/AAC.45.1.319-323.2001

Giovanetti, E., Brenciani, A., Burioni, R., \& Varaldo, P. E. (2002). A novel efflux system in inducibly erythromycin-resistant strains of Streptococcus pyogenes. Antimicrobial Agents and Chemotherapy, 46, 3750-3755. http://dx.doi.org/10.1128/AAC.46.12.3750-3755.2002

Haanpera, M., Huovinen, P., \& Jalava, J. (2005). Detection and quantification of macrolide resistance mutations at positions 2058 and 2059 of the 23S rRNE gene by pyrosequencing. Antimicrobial Agents and Chemotherapy, 49, 457-460 http://dx.doi.org/10.1128/AAC.49.1.457-460

Jalava, J., Vaara, M., \& Huovinen, P. (2004). Mutation at the position 2058 of the 23S rRNA as a cause of macrolide resistance in Streptococcus pyogenes. Annals of Clinical Microbiology and Antimicrobials, 3, 5-10. http://dx.doi.org/10.1186/1476-0711-3-5

Liu, S. L., Hessel, A., \& Sanderson, K. E. (1993). Genomic mapping with I-CeuI, an intron-encoded endonuclease specific for genes for ribosomal RNA, in Salmonella spp., Escherichia coli, and other bacteria. Proceedings of the National Academy of Sciences USA, 90, 6874-6878. http://dx.doi.org/10.1073/pnas.90.14.6874

Ludwig, W., Kirchof, N., Klugbauer, N., Weizenegger, M., Betzl, D., Ehrman, M., ... Wallnoef, P. R. (1992). Complete 23S ribosomal RNA sequences of gram-positive bacteria with a low $\mathrm{G}+\mathrm{C}$ content. Systematic and Applied Microbiology, 15, 487-501. http://dx.doi.org/10.1016/S0723-2020(11)80107-4

Pihlajamaki, M., Kataja, J., Seppala, H., Elliot, J., Leinonen, M., Huovinen, P., \& Jalava, J. (2002). Ribosomal mutations in Streptococcus pneumoniae clinical isolates. Antimicrobial Agents and Chemotherapy, 46, 654-658. http://dx.doi.org/10.1128/AAC.46.3.654-658.2002

Somkuti, G. A., \& Steinberg, D. H. (1986). Distribution and analysis of plasmids in Streptococcus thermophilus. Journal of Industrial Microbiology, 1, 157-163. http://dx.doi.org/10.1007/BF01569267

Sozzi, T., \& Smiley, M. B. (1980). Antibiotic resistance of yogurt cultures Streptococcus thermophilus and Lactobacillus bulgaricus. Applied and Environmental Microbiology, 40, 862-865.

Sutcliffe, J., Grebe, T., Tait-Kamradt, A., \& Wondrack, L. (1996). Detection of erythromycin-resistant determinants by PCR. Antimicrobial Agents and Chemotherapy, 40, 2562-2566.

Tait-Kamradt, A., Davies, T., Appelbaum, P. C., Depardieau, F., Courvalin, P., Petitpas, J., ... Sutcliffe, J. (2000). Two new mechanisms of macrolide resistance in clinical strains of Streptococcus pneumoniae from Eastern Europe and North America. Antimicrobial Agents and Chemotherapy, 44, 3395-3401. http://dx.doi.org/10.1128/AAC.44.12.3395-3401.2000 
Tosi, L., Berrutti, G., Danielsen, M., Wind, A., Huys, G., \& Morelli, L. (2007). Susceptibility of Streptococcus thermophiles to antibiotics. Antonie van Leeuwenhoek, 92, 21-28. http://dx.doi.org/10.1007/s10482-006-9130-6

Vester, B., \& Douthwaite, S. (2001). Macrolide resistance conferred by base substitutions in 23S rRNA. Antimicrobial Agents and Chemotherapy, 45, 1-12. http://dx.doi.org/10.1128/AAC.45.1.1-12.2001

Wand, H. H., Manuzon, M., Lehman, M., Wan, K., Luo, H., Wittum, T. E., ... Bakaletz, L. O. (2005). Food commensal microbes as a potentially important avenue in transmitting antibiotic resistance genes. FEMS Microbiology Letters, 255, 328-328.

\section{Copyrights}

Copyright for this article is retained by the author(s), with first publication rights granted to the journal.

This is an open-access article distributed under the terms and conditions of the Creative Commons Attribution license (http://creativecommons.org/licenses/by/3.0/). 